Pacific

Journal of

Mathematics

\title{
ESTIMATES OF THE GAPS BETWEEN CONSECUTIVE EIGENVALUES OF LAPLACIAN
}

Daguang Chen, Tao Zheng and HongCang Yang 


\title{
ESTIMATES OF THE GAPS BETWEEN CONSECUTIVE EIGENVALUES OF LAPLACIAN
}

\author{
Daguang Chen, TaO Zheng And HongCANG YANG
}

\begin{abstract}
For the eigenvalue problem of the Dirichlet Laplacian on a bounded domain in Euclidean space $\mathbb{R}^{n}$, we obtain estimates for the upper bounds of the gaps between consecutive eigenvalues which are the best possible in terms of the orders of the eigenvalues. Therefore, it is reasonable to conjecture that this type of estimate also holds for the eigenvalue problem on a Riemannian manifold. We give some particular examples.
\end{abstract}

\section{Introduction}

Let $\Omega$ be a bounded domain in an $n$-dimensional complete Riemannian manifold $M$ with boundary (possible empty). Then the Dirichlet eigenvalue problem of the Laplacian on $\Omega$ is given by

$$
\begin{cases}\Delta u=-\lambda u & \text { in } \Omega, \\ u=0 & \text { on } \partial \Omega,\end{cases}
$$

where $\Delta$ is the Laplacian on $M$. It is well known that the spectrum of (1-1) has the real and purely discrete eigenvalues

$$
0<\lambda_{1}<\lambda_{2} \leq \lambda_{3} \leq \cdots \nearrow \infty
$$

where each $\lambda_{i}$ has finite multiplicity and is repeated according to its multiplicity. The corresponding orthonormal basis of real eigenfunctions will be denoted $\left\{u_{j}\right\}_{j=1}^{\infty}$. We go forward under the assumption that $L^{2}(\Omega)$ represents the real Hilbert space of real-valued $L^{2}$ functions on $\Omega$. We put $\lambda_{0}=0$ if $\partial \Omega=\varnothing$.

An important aspect of estimating higher eigenvalues is to estimate as precisely as possible the gaps between consecutive eigenvalues of (1-1). In this regard, we will review some important results on the estimates of eigenvalue problem (1-1).

The work of Chen was partially supported by NSFC grants No. 11271213 and No. 11471180 . The work of Zheng was partially supported by the fundamental fund of Beijing Institute of Technology No. 20131742009 and No. 20141742002, postdoctoral fund No. 2014M550620 and No. 2015T80040, and NSFC grants No. 11401023 and No. 11471180. The work of Yang was partially supported by NSFC grant No. 11471180 and SF of CAS.

MSC2010: primary 35P15, 58C40; secondary 58J50.

Keywords: Laplacian, consecutive eigenvalues, test function, Riemannian manifold, hyperbolic space. 
For the upper bound of the gap between consecutive eigenvalues of (1-1), when $\Omega$ is a bounded domain in a 2-dimensional Euclidean space $\mathbb{R}^{2}$, Payne, Pólya and Weinberger (see [Payne et al. 1955; 1956]) proved

$$
\lambda_{k+1}-\lambda_{k} \leq \frac{2}{k} \sum_{i=1}^{k} \lambda_{i}
$$

C. J. Thompson [1969] extended (1-3) to the $n$-dimensional case and obtained

$$
\lambda_{k+1}-\lambda_{k} \leq \frac{4}{n k} \sum_{i=1}^{k} \lambda_{i}
$$

Hile and Protter [1980] improved (1-4) to

$$
\sum_{i=1}^{k} \frac{\lambda_{i}}{\lambda_{k+1}-\lambda_{i}} \geq \frac{n k}{4}
$$

Yang (see [Yang 1991] and more recently [Cheng and Yang 2007]) has obtained a sharp inequality:

$$
\sum_{i=1}^{k}\left(\lambda_{k+1}-\lambda_{i}\right)\left(\lambda_{k+1}-\left(1+\frac{4}{n}\right) \lambda_{i}\right) \leq 0
$$

From (1-6), one can infer

$$
\lambda_{k+1} \leq \frac{1}{k}\left(1+\frac{4}{n}\right) \sum_{i=1}^{k} \lambda_{i}
$$

The inequalities (1-6) and (1-7) are called Yang's first inequality and second inequality, respectively (see [Ashbaugh 1999; 2002; Ashbaugh and Benguria 1996; Harrell and Stubbe 1997]). Also we note that Ashbaugh and Benguria gave an optimal estimate for $k=1$ (see [Ashbaugh and Benguria 1991; 1992a; 1992b]). From Chebyshev's inequality, it is easy to prove that

$$
(1-6) \Longrightarrow(1-7) \Longrightarrow(1-5) \Longrightarrow(1-4) \text {. }
$$

From (1-6), Cheng and Yang [2005] obtained

$$
\lambda_{k+1}-\lambda_{k} \leq 2\left(\left(\frac{2}{n} \frac{1}{k} \sum_{i=1}^{k} \lambda_{i}\right)^{2}-\left(1+\frac{4}{n}\right) \frac{1}{k} \sum_{i=1}^{k}\left(\lambda_{i}-\frac{1}{k} \sum_{j=1}^{k} \lambda_{j}\right)^{2}\right)^{\frac{1}{2}} .
$$

Cheng and Yang [2007], using their recursive formula, obtained

$$
\lambda_{k+1} \leq C_{0}(n) k^{2 / n} \lambda_{1},
$$


where $C_{0}(n) \leq 1+4 / n$ is a constant. From Weyl's asymptotic formula (see [Weyl 1912]), we know that the upper bound (1-9) is best possible in terms of the order on $k$.

For a complete Riemannian manifold $M$, from Nash's theorem [1956], there exists an isometric immersion

$$
\psi: M \rightarrow \mathbb{R}^{N}
$$

where $\mathbb{R}^{N}$ is Euclidean space. The mean curvature of the immersion $\psi$ is denoted by $H$ and $|H|$ denotes its norm. Define

$$
\Phi=\{\psi \mid \psi \text { is an isometric immersion from } M \text { into Euclidean space }\} .
$$

When $\Omega$ is a bounded domain of a complete Riemannian manifold $M$, isometrically immersed into a Euclidean space $\mathbb{R}^{N}$, Cheng and the first author [Chen and Cheng 2008] (see also [El Soufi et al. 2009; Harrell 2007]) obtained

$$
\sum_{i=1}^{k}\left(\lambda_{k+1}-\lambda_{i}\right)^{2} \leq \frac{4}{n} \sum_{i=1}^{k}\left(\lambda_{k+1}-\lambda_{i}\right)\left(\lambda_{i}+\frac{1}{4} n^{2} H_{0}^{2}\right),
$$

where

$$
H_{0}^{2}=\inf _{\psi \in \Phi} \sup _{\Omega}|H|^{2}
$$

In the same paper, using the recursive formula in [Cheng and Yang 2007], Cheng and Chen also deduced

$$
\lambda_{k+1}+\frac{1}{4} n^{2} H_{0}^{2} \leq C_{0}(n) k^{2 / n}\left(\lambda_{1}+\frac{1}{4} n^{2} H_{0}^{2}\right),
$$

where $H_{0}^{2}$ and $C_{0}(n)$ are given by (1-11) and (1-9), respectively.

From (1-10), we can get the gaps between the consecutive eigenvalues of the Laplacian:

$$
\lambda_{k+1}-\lambda_{k} \leq 2\left(\left(\frac{2}{n} \frac{1}{k} \sum_{i=1}^{k} \lambda_{i}+\frac{1}{2} n H_{0}^{2}\right)^{2}-\left(1+\frac{4}{n}\right) \frac{1}{k} \sum_{i=1}^{k}\left(\lambda_{i}-\frac{1}{k} \sum_{j=1}^{k} \lambda_{j}\right)^{2}\right)^{\frac{1}{2}}
$$

Remark 1.1. When $\Omega$ is an $n$-dimensional compact homogeneous Riemannian manifold, a compact minimal submanifold without boundary and a connected bounded domain in the standard unit sphere $\mathbb{S}^{N}(1)$, and a connected bounded domain and a compact complex hypersurface without boundary of the complex projective space $\mathbb{C} \mathbb{P}^{n}(4)$ with holomorphic sectional curvature 4 , many mathematicians have studied the universal inequalities for eigenvalues and the difference of the consecutive eigenvalues (see [Cheng and Yang 2005; 2006; 2009; Harrell 1993; 
Harrell and Michel 1994; Harrell and Stubbe 1997; Li 1980; Yang and Yau 1980; Leung 1991; Sun et al. 2008; Chen et al. 2012]).

Remark 1.2. Another problem is the lower bound of the gap between the first two eigenvalues. In general, there exists the famous fundamental gap conjecture for the Dirichlet eigenvalue problem of the Schrödinger operator (see [Ashbaugh and Benguria 1989; van den Berg 1983; Singer et al. 1985; Yau 1986; Yu and Zhong 1986] and the references therein). The fundamental gap conjecture was solved by B. Andrews and J. Clutterbuck [2011].

From (1-8) and (1-13), it is not difficult to see that both Yang's estimate for the gap between consecutive eigenvalues of (1-1) implicit in [Yang 1991] and the estimate from [Chen and Cheng 2008] are on the order of $k^{3 /(2 n)}$. However, by the calculation of the gap between the consecutive eigenvalues of $\mathbb{S}^{n}$ with the standard metric and Weyl's asymptotic formula, the order of the upper bound of this gap is $k^{1 / n}$. Therefore, we make a conjecture:

Conjecture 1.3. Let $\Omega$ be a bounded domain in an n-dimensional complete Riemannian manifold $M$. For the Dirichlet problem (1-1), the upper bound for the gap between consecutive eigenvalues of the Laplacian should be

$$
\lambda_{k+1}-\lambda_{k} \leq C_{n, \Omega} k^{1 / n}, \quad k>1,
$$

where $C_{n, \Omega}$ is a constant dependent on $\Omega$ itself and the dimension $n$.

Remark 1.4. The famous Payne-Pólya-Weinberger conjecture (see [Payne et al. 1955; 1956; Thompson 1969; Ashbaugh and Benguria 1993a; 1993b]) states that, when $M=\mathbb{R}^{n}$, for the Dirichlet eigenvalue problem (1-1), one should have

$$
\frac{\lambda_{k+1}}{\lambda_{k}} \leq\left.\frac{\lambda_{2}}{\lambda_{1}}\right|_{\mathbb{B}^{n}}=\left(\frac{j_{n / 2,1}}{j_{n / 2-1,1}}\right)^{2},
$$

where $\mathbb{B}^{n}$ is the $n$-dimensional unit ball in $\mathbb{R}^{n}$, and $j_{p, k}$ is the $k$-th positive zero of the Bessel function $J_{p}(t)$. From Weyl's asymptotic formula and (1-15), the order of the upper bound of the consecutive eigenvalues of (1-1) is $k^{2 / n}$. Therefore, Conjecture 1.3 reflects the distribution of eigenvalues from another point of view. From the order of the upper bound of the gap between the consecutive eigenvalues of $\mathbb{S}^{n}$, the estimate in (1-14) is best possible in terms of the order on $k$.

In the following, the constants $C_{n, \Omega}$ are allowed to be different in different cases.

When $\Omega$ is a bounded domain in $\mathbb{R}^{n}$, for the Dirichlet eigenvalue problem (1-1), we give an affirmative answer to Conjecture 1.3.

Theorem 1.5. Let $\Omega \subset \mathbb{R}^{n}$ be a bounded domain in Euclidean space $\mathbb{R}^{n}$ and $\lambda_{k}$ be the $k$-th $(k>1)$ eigenvalue of the Dirichlet eigenvalue problem (1-1). Then we have

$$
\lambda_{k+1}-\lambda_{k} \leq C_{n, \Omega} k^{1 / n},
$$


where $C_{n, \Omega}=4 \lambda_{1} \sqrt{C_{0}(n) / n}$ and $C_{0}(n)$ is given by (1-9).

It is reasonable to conjecture that this type of estimate also holds on a Riemannian manifold. We give some particular examples as follows.

Corollary 1.6. Let $\Omega \subset \mathbb{M}^{n}(-1)$ be a bounded domain in hyperbolic space $\mathbb{M}^{n}(-1)$, and $\lambda_{k}$ be the $k$-th $(k>1)$ eigenvalue of the Dirichlet eigenvalue problem (1-1). Then we have

$$
\lambda_{k+1}-\lambda_{k} \leq C_{n, \Omega} k^{1 / n},
$$

where $C_{n, \Omega}$ depends on $\Omega$ and the dimension $n$ and is given by

$$
C_{n, \Omega}=4\left(C_{0}(n)\left(\lambda_{1}-\frac{1}{4}(n-1)^{2}\right)\left(\lambda_{1}+\frac{1}{4} n^{2} H_{0}^{2}\right)\right)^{1 / 2},
$$

where $C_{0}(n)$ and $H_{0}^{2}$ are the same as in (1-12).

In fact, by the comparison theorem for the distance function in a Riemannian manifold, we have:

Corollary 1.7. Let $M$ be an $n$-dimensional $(n \geq 3)$ simply connected complete noncompact Riemannian manifold with sectional curvature Sec satisfying

$$
-a^{2} \leq \operatorname{Sec} \leq-b^{2}
$$

where $a$ and $b$ are constants with $0 \leq b \leq a$. Let $\Omega \subset M$ be a bounded domain of $M$ and $\lambda_{k}$ be the $k$-th $(k>1)$ eigenvalue of $(1-1)$. Then we have

$$
\lambda_{k+1}-\lambda_{k} \leq C_{n, \Omega} k^{1 / n},
$$

where $C_{n, \Omega}$ depends on $\Omega$ and the dimension $n$ and is given by

$$
C_{n, \Omega}=4\left(C_{0}(n)\left(\lambda_{1}-\frac{1}{4}(n-1)^{2} b^{2}+\frac{1}{4}\left(a^{2}-b^{2}\right)\right)\left(\lambda_{1}+\frac{1}{4} n^{2} H_{0}^{2}\right)\right)^{1 / 2},
$$

where $C_{0}(n)$ and $H_{0}^{2}$ are the same as in (1-12).

\section{Preliminaries}

In this section, we first recall some basic concepts and a theorem of Chapter 10 in [Kolmogorov and Fomin 1960], and then we prove a theorem which will be used in the next section.

Define

$$
\mathcal{H}^{\infty}=\left\{x=\left(x_{j}\right)_{j=1}^{\infty} \mid x_{j} \in \mathbb{R},\left(\sum_{j=1}^{\infty} x_{j}^{2}\right)^{\frac{1}{2}}<+\infty\right\}
$$

and

$$
\mathcal{H}^{2}=\left\{x=\left(x_{1}, x_{2}\right) \mid x_{1}, x_{2} \in \mathbb{R},\left(x_{1}^{2}+x_{2}^{2}\right)^{1 / 2}<+\infty\right\} .
$$


The inner product $\langle\cdot, \cdot\rangle_{\infty}$ on $\mathcal{H}^{\infty}$ is defined by

$$
\langle x, y\rangle_{\infty}=\sum_{j=1}^{\infty} x_{j} y_{j}, \quad \forall x=\left(x_{j}\right)_{j=1}^{\infty}, y=\left(y_{j}\right)_{j=1}^{\infty} .
$$

The inner product $\langle\cdot, \cdot\rangle_{2}$ on $\mathcal{H}^{2}$ can be defined similarly. Obviously, both $\mathcal{H}^{\infty}$ and $\mathcal{H}^{2}$ are Hilbert spaces. The dual space of $\mathcal{H}^{2}$ is denoted by $\left(\mathcal{H}^{2}\right)^{*}$. It is well known that $\left(\mathcal{H}^{2}\right)^{*}$ is isomorphic to $\mathcal{H}^{2}$ itself.

In order to prove our theorem, we need the following Lagrange multiplier theorem for real Banach spaces (see Chapter 10, Section 3, paragraph 3 in [Kolmogorov and Fomin 1960] or page 270 in [Zeidler 1995]).

Theorem 2.1. Let $X$ and $Y$ be real Banach spaces. Assume that $F: x_{0} \in U \subset X \rightarrow \mathbb{R}$ and $\Phi: x_{0} \in U \subset X \rightarrow Y$ are continuously Fréchet differentiable on an open neighborhood of $x_{0}$, where $x_{0} \in \Phi^{-1}(0)=\{x \in U \mid \Phi(x)=0 \in Y\}$. If the set $\left\{\Phi^{\prime}\left(x_{0}\right)(x) \in Y \mid x \in X\right\}$ is closed and $x_{0}$ is an extremum (maximum or minimum) of $F$ on $\Phi^{-1}(0)$, then there exists $\lambda_{0} \in \mathbb{R}$ and a linear functional $y^{*} \in Y^{*}$, where

$$
\lambda_{0}^{2}+\left\|y^{*}\right\|^{2} \neq 0
$$

such that

$$
\lambda_{0} F^{\prime}\left(x_{0}\right)+\left(\Phi^{\prime}\left(x_{0}\right)\right)^{*}\left(y^{*}\right)=0 .
$$

Moreover, if $\left\{\Phi^{\prime}\left(x_{0}\right)(x) \in Y \mid x \in X\right\}=Y$, then we can take $\lambda_{0}=1$.

Theorem 2.2. Assume that $\left\{\mu_{j}\right\}_{j=1}^{\infty}$ is a nondecreasing sequence, i.e.,

$$
0<\mu_{1} \leq \mu_{2} \leq \cdots \leq \mu_{k} \leq \cdots \nearrow \infty
$$

where each $\mu_{i}$ has finite multiplicity $m_{i}$ and is repeated according to its multiplicity.

Define

$$
\begin{aligned}
& B=\sum_{j=1}^{\infty} x_{j}^{2}>0, \\
& A=\sum_{j=1}^{\infty} \mu_{j}^{2} x_{j}^{2}, \quad x=\left(x_{j}\right)_{j=1}^{\infty} \in \mathcal{H}^{\infty} .
\end{aligned}
$$

If $x_{m_{1}} \neq 0$ and $\sum_{j=1}^{\infty} \mu_{j} x_{j}^{2}<\sqrt{A B}$, under the conditions in (2-2), we have

$$
\sum_{j=1}^{\infty} \mu_{j} x_{j}^{2} \leq \frac{A+\mu_{m_{1}} \mu_{m_{1}+1} B}{\mu_{m_{1}}+\mu_{m_{1}+1}} .
$$


Proof. First, assume that $\left\{\mu_{j}\right\}_{j=1}^{\infty}$ is a strictly increasing sequence, i.e.,

$$
0<\mu_{1}<\mu_{2}<\cdots<\mu_{k}<\cdots \nearrow \infty \text {. }
$$

Suppose

$$
\begin{aligned}
& F(x)=\sum_{j=1}^{\infty} \mu_{j} x_{j}^{2}, \\
& \Psi(x)=\left(\sum_{j=1}^{\infty} x_{j}^{2}-B, \sum_{j=1}^{\infty} \mu_{j}^{2} x_{j}^{2}-A\right) \in \mathcal{H}^{2}, \quad x \in \mathcal{H}^{\infty} .
\end{aligned}
$$

Let $x_{0}=\left(a_{j}\right)_{j=1}^{\infty}$ be an extremum of $F(x)$ on $\Phi^{-1}(0)$. Since $\forall h=\left(h_{j}\right)_{j=1}^{\infty} \in \mathcal{H}^{\infty}$,

$$
\begin{gathered}
F^{\prime}\left(x_{0}\right) h=2 \sum_{j=1}^{\infty} \mu_{j} x_{j} h_{j}, \\
\Psi^{\prime}\left(x_{0}\right) h=\left(2 \sum_{j=1}^{\infty} x_{j} h_{j}, 2 \sum_{j=1}^{\infty} \mu_{j}^{2} x_{j} h_{j}\right),
\end{gathered}
$$

and

$$
\Psi^{\prime}\left(x_{0}\right)\left(\mathcal{H}^{\infty}\right)=\mathcal{H}^{2},
$$

there exists $y^{*} \in\left(\mathcal{H}^{2}\right)^{*}$ such that

$$
F^{\prime}\left(x_{0}\right) h+\left(\Psi^{\prime}\left(x_{0}\right)\right)^{*}\left(y^{*}\right) h=0 .
$$

Since $\mathcal{H}^{2}=\left(\mathcal{H}^{2}\right)^{*}$, we can use some unique vector $(\mu, \lambda) \in \mathcal{H}^{2}$ to rewrite $(2-4)$ as

$$
\sum_{j=1}^{\infty} \mu_{j} a_{j} h_{j}+\mu \sum_{j=1}^{\infty} a_{j} h_{j}+\lambda \sum_{j=1}^{\infty} \mu_{j}^{2} a_{j} h_{j}=0 .
$$

Choosing

$$
h_{j}=\delta_{j k}, \quad j=1,2, \ldots,
$$

from (2-5), we obtain a system of equations

$$
\mu_{k} a_{k}+\mu a_{k}+\lambda \mu_{k}^{2} a_{k}=0, \quad k=1,2, \ldots
$$

Since $\left\{\mu_{k}\right\}$ is a strictly increasing sequence, and there are only two varieties $\mu$ and $\lambda$, there are only two cases for $x_{0}$.

Case 1. There is only one $a_{k} \neq 0$, whether $k=1$ or not. In this case, the critical value of $F(x)$ is given by

$$
F\left(x_{0}\right)=\sqrt{A B},
$$

which contradicts the assumption of the theorem. 
Case 2. There are only two nonzero components of $x_{0}$, say $a_{k}$ and $a_{l}$ (without loss of generality, set $k<l$ ). In this case, we have

$$
\begin{aligned}
& A=\mu_{k}^{2} a_{k}^{2}+\mu_{l}^{2} a_{l}^{2}, \\
& B=a_{k}^{2}+a_{l}^{2} .
\end{aligned}
$$

From (2-7), we have

$$
F\left(x_{0}\right)=\frac{A+\mu_{k} \mu_{l} B}{\mu_{k}+\mu_{l}} .
$$

Since

$$
A=\mu_{k}^{2} a_{k}^{2}+\mu_{l}^{2} a_{l}^{2}>\mu_{k}^{2}\left(a_{k}^{2}+a_{l}^{2}\right)=\mu_{k}^{2} B
$$

we have

$$
\mu_{k}<\sqrt{A / B}
$$

Similarly, we can also deduce

$$
\mu_{l}>\sqrt{A / B}
$$

Hence, we have

$$
F\left(x_{0}\right)-\sqrt{A B}=\frac{B\left(\mu_{k}-\sqrt{A / B}\right)\left(\mu_{l}-\sqrt{A / B}\right)}{\mu_{k}+\mu_{l}}<0 .
$$

Since $\left\{\mu_{i}\right\}$ is a strictly increasing sequence, for $\mu_{k}$ fixed, from (2-8) and (2-9), we know that the right side of (2-10) is strictly decreasing in $\mu_{l}$, i.e.,

$$
\frac{B\left(\mu_{k}-\sqrt{A / B}\right)\left(\mu_{k+1}-\sqrt{A / B}\right)}{\mu_{k}+\mu_{k+1}}>\frac{B\left(\mu_{k}-\sqrt{A / B}\right)\left(\mu_{k+2}-\sqrt{A / B}\right)}{\mu_{k}+\mu_{k+2}}>\cdots .
$$

Hence, we know that

$$
\frac{A+\mu_{k} \mu_{k+1} B}{\mu_{k}+\mu_{k+1}}, \quad k=1,2, \ldots,
$$

are local maximal values of $F(x)$.

Since $x_{m_{1}}=x_{1} \neq 0, k$ must be equal to $m_{1}=1$ only. Finally, we have the global maximum of $F(x)$

$$
\frac{A+\mu_{1} \mu_{2} B}{\mu_{1}+\mu_{2}} .
$$

Second, assume that $\left\{\mu_{j}\right\}_{j=1}^{\infty}$ is an increasing sequence, i.e.,

$$
0<\mu_{1} \leq \mu_{2} \leq \cdots \leq \mu_{k} \leq \cdots \nearrow \infty
$$

where each $\mu_{i}$ has finite multiplicity $m_{i}$ and is repeated according to its multiplicity. 
Replacing (2-7) by

$$
\begin{aligned}
& A=m_{k} \mu_{k}^{2} a_{k}^{2}+m_{l} \mu_{l}^{2} a_{l}^{2}, \\
& B=m_{k} a_{k}^{2}+m_{l} a_{l}^{2},
\end{aligned}
$$

and following the above steps almost word for word, we deduce that the local maximal value of $F(x)$ is

$$
\frac{A+\mu_{m_{k}} \mu_{m_{k}+1} B}{\mu_{m_{k}}+\mu_{m_{k}+1}}
$$

and

$$
\mu_{m_{k}}<\sqrt{A / B}, \quad \mu_{m_{k}+1}>\sqrt{A / B} .
$$

Since $x_{m_{1}} \neq 0, m_{k}$ must be equal to $m_{1}$ and the local maximal value of $F(x)$ is the global maximum. Since

$$
\frac{A+\mu_{m_{1}} \mu_{m_{1}+1} B}{\mu_{m_{1}}+\mu_{m_{1}+1}}-\sqrt{A B}=\frac{B\left(\mu_{m_{1}}-\sqrt{A / B}\right)\left(\mu_{m_{k}+1}-\sqrt{A / B}\right)}{\mu_{m_{1}}+\mu_{m_{1}+1}}<0,
$$

we can obtain (2-3).

\section{Proofs of main results}

In this section, we will give the proof of Theorem 1.5. In order to prove our main results, we need the following key lemma and related corollaries of Theorem 2.2.

Lemma 3.1. For the Dirichlet eigenvalue problem (1-1), let $u_{k}$ be the orthonormal eigenfunction corresponding to the $k$-th eigenvalue $\lambda_{k}$, i.e.,

$$
\begin{cases}\Delta u_{k}=-\lambda_{k} u_{k} & \text { in } \Omega, \\ u_{k}=0 & \text { on } \partial \Omega, \\ \int_{\Omega} u_{i} u_{j}=\delta_{i j} . & \end{cases}
$$

Then for any complex-valued function $g \in C^{3}(\Omega) \cap C^{2}(\bar{\Omega})$ such that $g u_{i}$ is not the $\mathbb{C}$-linear combination of

$$
u_{1}, \ldots, u_{k+1} \text {, }
$$

and such that

$$
a_{k+1}=\int_{\Omega} g u_{i} u_{k+1} \neq 0,
$$

with $\lambda_{i}<\lambda_{k+1}<\lambda_{k+2}, k, i \in \mathbb{Z}^{+}, i \geq 1$, we have

$$
\begin{aligned}
\left(\left(\lambda_{k+1}-\lambda_{i}\right)\right. & \left.+\left(\lambda_{k+2}-\lambda_{i}\right)\right) \int_{\Omega}|\nabla g|^{2} u_{i}^{2} \\
& \leq \int_{\Omega}\left|2 \nabla g \cdot \nabla u_{i}+u_{i} \Delta g\right|^{2}+\left(\lambda_{k+1}-\lambda_{i}\right)\left(\lambda_{k+2}-\lambda_{i}\right) \int_{\Omega}\left|g u_{i}\right|^{2} .
\end{aligned}
$$


Proof. Define

$$
\begin{aligned}
a_{i j} & =\int_{\Omega} g u_{i} u_{j}, \\
b_{i j} & =\int_{\Omega}\left(\nabla u_{i} \cdot \nabla g+\frac{1}{2} u_{i} \Delta g\right) u_{j},
\end{aligned}
$$

where $\nabla$ denotes the gradient operator. Obviously,

$$
a_{i j}=a_{j i} .
$$

Then, from Stokes' theorem, we get

$$
\begin{aligned}
\lambda_{j} a_{i j} & =\int_{\Omega} g u_{i}\left(-\Delta u_{j}\right) \\
& =-\int_{\Omega}\left(u_{i} \Delta g+g \Delta u_{i}+2 \nabla g \cdot \nabla u_{i}\right) u_{j} \\
& =\lambda_{i} \int_{\Omega} g u_{i} u_{j}-2 \int_{\Omega}\left(\nabla u_{i} \cdot \nabla g+\frac{1}{2} u_{i} \Delta g\right) u_{j},
\end{aligned}
$$

i.e.,

$$
2 b_{i j}=\left(\lambda_{i}-\lambda_{j}\right) a_{i j}
$$

From Stokes' theorem, we have

$$
\int_{\Omega}|\nabla g|^{2} u_{i}^{2}=-2 \int_{\Omega} g u_{i}\left(\nabla \bar{g} \cdot \nabla u_{i}+\frac{1}{2} u_{i} \Delta \bar{g}\right) .
$$

Since $\left\{u_{k}\right\}_{k=1}^{\infty}$ consists of a complete orthonormal basis of $L^{2}(\Omega)$, by the definition of $a_{i j}$ and $b_{i j}$, from (3-3), (3-4) and Parseval's identity, we obtain

$$
\begin{gathered}
\int_{\Omega}\left|g u_{i}\right|^{2}=\sum_{j=1}^{\infty}\left|a_{i j}\right|^{2}, \\
\int_{\Omega}|\nabla g|^{2} u_{i}^{2}=2 \sum_{j=1}^{\infty} a_{i j} \overline{b_{i j}}=\sum_{j=1}^{\infty}\left(\lambda_{j}-\lambda_{i}\right)\left|a_{i j}\right|^{2}, \\
\int_{\Omega}\left|2 \nabla \bar{g} \cdot \nabla u_{i}+u_{i} \Delta \bar{g}\right|^{2}=4 \sum_{j=1}^{\infty}\left|b_{i j}\right|^{2}=\sum_{j=1}^{\infty}\left(\lambda_{j}-\lambda_{i}\right)^{2}\left|a_{i j}\right|^{2} .
\end{gathered}
$$

From the Cauchy-Schwarz inequality, we have

$$
\left(\sum_{j=k+1}^{\infty}\left(\lambda_{j}-\lambda_{i}\right)\left|a_{i j}\right|^{2}\right)^{2} \leq \sum_{j=k+1}^{\infty}\left(\lambda_{j}-\lambda_{i}\right)^{2}\left|a_{i j}\right|^{2} \sum_{j=k+1}^{\infty}\left|a_{i j}\right|^{2} .
$$


From (3-5), (3-6), (3-7) and (3-8), we can deduce

$$
\begin{aligned}
& \left(\int_{\Omega}|\nabla g|^{2} u_{i}^{2}-\sum_{j=1}^{k}\left(\lambda_{j}-\lambda_{i}\right)\left|a_{i j}\right|^{2}\right)^{2} \\
& \leq\left(\int_{\Omega}\left|g u_{i}\right|^{2}-\sum_{j=1}^{k}\left|a_{i j}\right|^{2}\right)\left(\int_{\Omega}\left|2 \nabla g \cdot \nabla u_{i}+u_{i} \Delta g\right|^{2}-\sum_{j=1}^{k}\left(\lambda_{j}-\lambda_{i}\right)^{2}\left|a_{i j}\right|^{2}\right) .
\end{aligned}
$$

Define

$$
\begin{aligned}
\widetilde{B}(i) & =\int_{\Omega}\left|g u_{i}\right|^{2}-\sum_{j=1}^{k}\left|a_{i j}\right|^{2}=\sum_{j=k+1}^{\infty}\left|a_{i j}\right|^{2}>0, \quad \text { since } \int_{\Omega} g u_{i} u_{k+1} \neq 0, \\
\widetilde{A}(i) & =\int_{\Omega}\left|2 \nabla g \cdot \nabla u_{i}+u_{i} \Delta g\right|^{2}-\sum_{j=1}^{k}\left(\lambda_{j}-\lambda_{i}\right)^{2}\left|a_{i j}\right|^{2} \\
& =\sum_{j=k+1}^{\infty}\left(\lambda_{j}-\lambda_{i}\right)^{2}\left|a_{i j}\right|^{2} \geq 0, \\
\widetilde{C}(i) & =\int_{\Omega}|\nabla g|^{2} u_{i}^{2}-\sum_{j=1}^{k}\left(\lambda_{j}-\lambda_{i}\right)\left|a_{i j}\right|^{2}=\sum_{j=k+1}^{\infty}\left(\lambda_{j}-\lambda_{i}\right)\left|a_{i j}\right|^{2} .
\end{aligned}
$$

Since $g u_{i}$ is not the $\mathbb{C}$-linear combination of

$$
u_{1}, \ldots, u_{k+1}
$$

there exists some $l>k+1$ such that

$$
a_{l}=\int_{\Omega} g u_{i} u_{l} \neq 0
$$

Since

$$
\lambda_{i}<\lambda_{k+1}<\lambda_{k+2} \leq \lambda_{l},
$$

the vector

$$
\left(\left|a_{i j}\right|\right)_{j=k+1}^{\infty}
$$

is not proportional to

$$
\left(\left(\lambda_{j}-\lambda_{i}\right)^{2}\left|a_{i j}\right|\right)_{j=k+1}^{\infty} .
$$

From the Cauchy-Schwarz inequality, we have

$$
\widetilde{C}(i)<\sqrt{\widetilde{A}(i) \widetilde{B}(i)} .
$$

Since $a_{k+1} \neq 0$, from (3-10) and Theorem 2.2, we have

$$
\widetilde{C}(i) \leq \frac{\widetilde{A}(i)+\left(\lambda_{k+2}-\lambda_{i}\right)\left(\lambda_{k+1}-\lambda_{i}\right) \widetilde{B}(i)}{\left(\lambda_{k+2}-\lambda_{i}\right)+\left(\lambda_{k+1}-\lambda_{i}\right)} .
$$


From (3-11) and the definition of $\widetilde{A}(i), \widetilde{B}(i)$ and $\widetilde{C}(i)$, we obtain

$$
\begin{gathered}
\left(\left(\lambda_{k+2}-\lambda_{i}\right)+\left(\lambda_{k+1}-\lambda_{i}\right)\right) \int_{\Omega}|\nabla g|^{2} u_{i}^{2} \\
\leq \int_{\Omega}\left|2 \nabla g \cdot \nabla u_{i}+u_{i} \Delta g\right|^{2}+\left(\lambda_{k+1}-\lambda_{i}\right)\left(\lambda_{k+2}-\lambda_{i}\right) \int_{\Omega}\left|g u_{i}\right|^{2} \\
\quad-\sum_{j=1}^{k}\left(\lambda_{k+1}-\lambda_{j}\right)\left(\lambda_{k+2}-\lambda_{j}\right)\left|a_{i j}\right|^{2} \\
\leq \int_{\Omega}\left|2 \nabla g \cdot \nabla u_{i}+u_{i} \Delta g\right|^{2}+\left(\lambda_{k+1}-\lambda_{i}\right)\left(\lambda_{k+2}-\lambda_{i}\right) \int_{\Omega}\left|g u_{i}\right|^{2} .
\end{gathered}
$$

Corollary 3.2. Under the assumption of Lemma 3.1, for any nonconstant realvalued function $f \in C^{3}(\Omega) \cap C^{2}(\bar{\Omega})$, we have

$$
\begin{aligned}
& \left(\left(\lambda_{k+2}-\lambda_{i}\right)+\left(\lambda_{k+1}-\lambda_{i}\right)\right) \int_{\Omega}|\nabla f|^{2} u_{i}^{2} \\
& \quad \leq 2 \sqrt{\left(\left(\lambda_{k+2}-\lambda_{i}\right)\left(\lambda_{k+1}-\lambda_{i}\right)\right) \int_{\Omega}|\nabla f|^{4} u^{2}}+\int_{\Omega}\left(2 \nabla f \cdot \nabla u_{i}+u_{i} \Delta f\right)^{2} .
\end{aligned}
$$

Proof. Taking $g=\exp (\sqrt{-1} \alpha f), \alpha \in \mathbb{R} \backslash\{0\}$, in (3-1), we have

$$
\begin{aligned}
& \alpha^{2}\left(\left(\lambda_{k+1}-\lambda_{i}\right)+\left(\lambda_{k+2}-\lambda_{i}\right)\right) \int_{\Omega}|\nabla f|^{2} u_{i}^{2} \\
& \quad \leq \alpha^{4} \int_{\Omega}|\nabla f|^{4} u_{i}^{2}+\alpha^{2} \int_{\Omega}\left|2 \nabla f \cdot \nabla u_{i}+u_{i} \Delta f\right|^{2}+\left(\lambda_{k+1}-\lambda_{i}\right)\left(\lambda_{k+2}-\lambda_{i}\right) .
\end{aligned}
$$

From (3-14), we have

$$
\begin{aligned}
& \left(\left(\lambda_{k+1}-\lambda_{i}\right)+\left(\lambda_{k+2}-\lambda_{i}\right)\right) \int_{\Omega}|\nabla f|^{2} u_{i}^{2} \\
& \leq \alpha^{2} \int_{\Omega}|\nabla f|^{4} u_{i}^{2}+\frac{1}{\alpha^{2}}\left(\lambda_{k+1}-\lambda_{i}\right)\left(\lambda_{k+2}-\lambda_{i}\right)+\int_{\Omega}\left|2 \nabla f \cdot \nabla u_{i}+u_{i} \Delta f\right|^{2} .
\end{aligned}
$$

Since the inequality (3-15) is valid for any $\alpha \neq 0$ and

$$
\left(\lambda_{k+1}-\lambda_{i}\right)\left(\lambda_{k+2}-\lambda_{i}\right) \neq 0, \quad \int_{\Omega}|\nabla f|^{4} u_{i}^{2} \neq 0,
$$

we can choose

$$
\alpha^{2}=\left(\frac{\left(\lambda_{k+1}-\lambda_{i}\right)\left(\lambda_{k+2}-\lambda_{i}\right)}{\int_{\Omega}|\nabla f|^{4} u_{i}^{2}}\right)^{\frac{1}{2}}
$$

to have (3-13). 
Corollary 3.3. Under the assumption of Lemma 3.1, for any real-valued function $f \in C^{3}(\Omega) \cap C^{2}(\bar{\Omega})$ with $|\nabla f|^{2}=1$, we have

$$
\begin{aligned}
& \left(\lambda_{k+2}-\lambda_{k+1}\right)^{2} \\
& \quad \leq 16\left(\int_{\Omega}\left(\nabla f \cdot \nabla u_{i}\right)^{2}-\frac{1}{4} \int_{\Omega}(\Delta f)^{2} u_{i}^{2}-\frac{1}{2} \int_{\Omega}(\nabla(\Delta f) \cdot \nabla f) u_{i}^{2}\right) \lambda_{k+2} .
\end{aligned}
$$

Furthermore, we have

$$
\lambda_{k+2}-\lambda_{k+1} \leq 4\left(\lambda_{i}-\frac{1}{4} \int_{\Omega}(\Delta f)^{2} u_{i}^{2}-\frac{1}{2} \int_{\Omega}(\nabla(\Delta f) \cdot \nabla f) u_{i}^{2}\right)^{\frac{1}{2}} \sqrt{\lambda_{k+2}} .
$$

Proof. From Corollary 3.2 and $|\nabla f|^{2}=1$, we have

$$
\left(\left(\lambda_{k+2}-\lambda_{i}\right)+\left(\lambda_{k+1}-\lambda_{i}\right)\right)-2 \sqrt{\left(\lambda_{k+2}-\lambda_{i}\right)\left(\lambda_{k+1}-\lambda_{i}\right)} \leq \int_{\Omega}\left(2 \nabla f \cdot \nabla u_{i}+u_{i} \Delta f\right)^{2},
$$

that is,

$$
\left(\sqrt{\lambda_{k+2}-\lambda_{i}}-\sqrt{\lambda_{k+1}-\lambda_{i}}\right)^{2} \leq \int_{\Omega}\left(2 \nabla f \cdot \nabla u_{i}+u_{i} \Delta f\right)^{2} .
$$

By integration by parts, we have

$$
\int_{\Omega}\left(2 \nabla f \cdot \nabla u_{i}+u_{i} \Delta f\right)^{2}=4 \int_{\Omega}\left(\nabla f \cdot \nabla u_{i}\right)^{2}-\int_{\Omega}(\Delta f)^{2} u_{i}^{2}-2 \int_{\Omega}(\nabla(\Delta f) \cdot \nabla f) u_{i}^{2} .
$$

Hence, we have

$$
\begin{aligned}
\left(\sqrt{\lambda_{k+2}-\lambda_{i}}-\right. & \left.\sqrt{\lambda_{k+1}-\lambda_{i}}\right)^{2} \\
& \leq 4 \int_{\Omega}\left(\nabla f \cdot \nabla u_{i}\right)^{2}-\int_{\Omega}(\Delta f)^{2} u_{i}^{2}-2 \int_{\Omega}(\nabla(\Delta f) \cdot \nabla f) u_{i}^{2} .
\end{aligned}
$$

Multiplying (3-18) by $\left(\sqrt{\lambda_{k+2}-\lambda_{i}}+\sqrt{\lambda_{k+1}-\lambda_{i}}\right)^{2}$ on both sides, we can get

$$
\begin{aligned}
\left(\lambda_{k+2}-\lambda_{k+1}\right)^{2} \leq & 4\left(\int_{\Omega}\left(\nabla f \cdot \nabla u_{i}\right)^{2}-\frac{1}{4} \int_{\Omega}(\Delta f)^{2} u_{i}^{2}-\frac{1}{2} \int_{\Omega}(\nabla(\Delta f) \cdot \nabla f) u_{i}^{2}\right) \\
& \times\left(\sqrt{\lambda_{k+2}-\lambda_{i}}+\sqrt{\lambda_{k+1}-\lambda_{i}}\right)^{2} \\
& \leq 16\left(\int_{\Omega}\left(\nabla f \cdot \nabla u_{i}\right)^{2}-\frac{1}{4} \int_{\Omega}(\Delta f)^{2} u_{i}^{2}-\frac{1}{2} \int_{\Omega}(\nabla(\Delta f) \cdot \nabla f) u_{i}^{2}\right) \lambda_{k+2},
\end{aligned}
$$

which is the inequality (3-16). 
Since $|\nabla f|^{2}=1$, from (3-16), the Cauchy-Schwarz inequality and integration by parts, we obtain

$$
\begin{aligned}
\left(\lambda_{k+2}-\lambda_{k+1}\right)^{2} & \leq 16\left(\int_{\Omega}\left|\nabla u_{i}\right|^{2}-\frac{1}{4} \int_{\Omega}(\Delta f)^{2} u_{i}^{2}-\frac{1}{2} \int_{\Omega}(\nabla(\Delta f) \cdot \nabla f) u_{i}^{2}\right) \lambda_{k+2} \\
& =16\left(\lambda_{i}-\frac{1}{4} \int_{\Omega}(\Delta f)^{2} u_{i}^{2}-\frac{1}{2} \int_{\Omega}(\nabla(\Delta f) \cdot \nabla f) u_{i}^{2}\right) \lambda_{k+2} .
\end{aligned}
$$

Remark 3.4. If $\lambda_{k+1}=\lambda_{k+2}$, (3-17) also holds trivially. Hence, under the conditions in Corollary 3.3, when $i=1$, (3-17) holds for any $k>1$.

Proof of Theorem 1.5. Since the inequality (3-20) always holds for $\lambda_{k+1}=\lambda_{k+2}$, without loss of generality, we assume that $\lambda_{k+1}<\lambda_{k+2}$ in the following discussion.

Let $\left\{x_{1}, x_{2}, \ldots, x_{n}\right\}$ be the standard coordinate functions in $\mathbb{R}^{n}$. Taking

$$
i=1 \quad \text { and } \quad f=x_{l}, \quad l=1, \ldots, n,
$$

in (3-16) and summing over $l$ from 1 to $n$, we have

$$
n\left(\lambda_{k+2}-\lambda_{k+1}\right)^{2} \leq 16 \lambda_{k+2} \int_{\Omega} \sum_{l=1}^{n}\left(\frac{\partial u_{1}}{\partial x_{l}}\right)^{2}=16 \lambda_{1} \lambda_{k+2},
$$

where we use $\left|\nabla x_{l}\right|=1, l=1, \ldots, n$.

From Theorem 3.1 in [Cheng and Yang 2007] (see also (1-9)) and from (3-19), we deduce

$$
\lambda_{k+2}-\lambda_{k+1} \leq 4 \sqrt{\frac{\lambda_{1}}{n}} \sqrt{\lambda_{k+2}} \leq 4 \lambda_{1} \sqrt{\frac{C_{0}(n)}{n}}(k+1)^{1 / 2}=C_{n, \Omega}(k+1)^{1 / 2},
$$

where $C_{n, \Omega}=4 \lambda_{1} \sqrt{C_{0}(n) / n}$ and $C_{0}(n)$ is given by (1-9).

Therefore, (3-20) holds for arbitrary $k>1$.

Proof of Corollary 1.6. For convenience, we will use the upper-half-plane model of hyperbolic space, i.e.,

$$
\mathbb{H}^{n}(-1)=\left\{\left(x_{1}, \ldots, x_{n}\right) \in \mathbb{R}^{n} \mid x_{n}>0\right\}
$$

with the standard metric

$$
\mathrm{d} s^{2}=\frac{\left(\mathrm{d} x_{1}\right)^{2}+\cdots+\left(\mathrm{d} x_{n}\right)^{2}}{\left(x_{n}\right)^{2}} .
$$

Taking $r=\log x_{n}$, we have

$$
\mathrm{d} s^{2}=(\mathrm{d} r)^{2}+\mathrm{e}^{-2 r} \sum_{i=1}^{n-1}\left(\mathrm{~d} x_{i}\right)^{2}
$$


Without loss of generality, we assume that $\lambda_{k+1}<\lambda_{k+2}$. Taking $f=r$ and $i=1$ in (3-17), we have

$$
\begin{aligned}
\lambda_{k+2}-\lambda_{k+1} & \leq 4\left(\lambda_{1}-\frac{1}{4} \int_{\Omega}(\Delta r)^{2} u_{i}^{2}-\frac{1}{2} \int_{\Omega}(\nabla(\Delta r) \cdot \nabla r) u_{1}^{2}\right)^{\frac{1}{2}} \sqrt{\lambda_{k+2}} \\
& =4\left(\lambda_{1}-\frac{1}{4}(n-1)^{2}\right)^{1 / 2} \sqrt{\lambda_{k+2}},
\end{aligned}
$$

where $|\nabla r|=1$ and $\Delta r=-(n-1)$ are used.

By (1-12) and (3-21), we have

$$
\begin{aligned}
\lambda_{k+2}-\lambda_{k+1} & \leq 4\left(\lambda_{1}-\frac{1}{4}(n-1)^{2}\right)^{1 / 2} \sqrt{C_{0}(n)\left(\lambda_{1}+\frac{1}{4} n^{2} H_{0}^{2}\right)}(k+1)^{1 / n} \\
& =C_{n, \Omega}(k+1)^{1 / n},
\end{aligned}
$$

where $C_{n, \Omega}$ is defined by (1-18). Therefore, we can deduce (3-22) for any $k>1$.

\section{Proof of Corollary 1.7}

Assume that $(M, g)$ is an $n$-dimensional complete noncompact Riemannian manifold with sectional curvature Sec satisfying $-a^{2} \leq \operatorname{Sec} \leq-b^{2}$, where $a$ and $b$ are constants with $0 \leq b \leq a$. Let $\Omega$ be a bounded domain of $M$. For a fixed point $p \notin \bar{\Omega}$, the distance function $\rho(x)$ is defined by $\rho(x)=\operatorname{distance}(x, p)$. From $|\nabla \rho|=1$ and Proposition 2.2 of [Schoen and Yau 1994], we have

$$
\nabla \rho \cdot \nabla(\Delta \rho)=-|\operatorname{Hess} \rho|^{2}-\operatorname{Ric}(\nabla \rho, \nabla \rho) .
$$

Assume that $h_{1}, \ldots, h_{n-1}$, with $0 \leq h_{1} \leq \cdots \leq h_{n-1}$, are the eigenvalues of Hess $\rho$. We have

$$
\begin{aligned}
2 \mid \text { Hess }\left.\rho\right|^{2}-(\Delta \rho)^{2} & =2 \sum_{i=1}^{n-1} h_{i}^{2}-\left(\sum_{i=1}^{n-1} h_{i}\right)^{2} \\
& =\sum_{i=1}^{n-1} h_{i}^{2}-\sum_{i \neq j} h_{i} h_{j} \\
& \leq h_{n-1}^{2}+h_{1} h_{2}+\cdots+h_{n-2} h_{n-1}-\sum_{i \neq j} h_{i} h_{j} \\
& =h_{n-1}^{2}-h_{1} h_{2}-\cdots-h_{n-2} h_{n-1}-\sum_{\substack{i \neq j \\
i, j \leq n-2}} h_{i} h_{j} \\
& \leq h_{n-1}^{2}-(n-2)^{2} h_{1}^{2} .
\end{aligned}
$$

From the Hessian comparison theorem (see [Wu et al. 1989]), we have

$$
a \frac{\cosh a \rho}{\sinh a \rho} \geq h_{n-1} \geq \cdots \geq h_{1} \geq b \frac{\cosh b \rho}{\sinh b \rho} .
$$


Since $n \geq 3$ and $a^{2} /\left(\sinh ^{2} a \rho\right)$ is a decreasing function of $a$, from (4-2) and (4-3), we have

$$
\begin{aligned}
2 \mid \text { Hess }\left.\rho\right|^{2} & +2 \operatorname{Ric}(\nabla \rho, \nabla \rho)-(\Delta \rho)^{2} \\
& \leq a^{2} \frac{\cosh ^{2} a \rho}{\sinh ^{2} a \rho}-(n-2)^{2} b^{2} \frac{\cosh ^{2} b \rho}{\sinh ^{2} b \rho}-2(n-1) b^{2} \\
& =a^{2}+\frac{a^{2}}{\sinh ^{2} a \rho}-(n-2)^{2} b^{2}-(n-2)^{2} \frac{b^{2}}{\sinh ^{2} b \rho}-2(n-1) b^{2} \\
& \leq-(n-1)^{2} b^{2}+\left(a^{2}-b^{2}\right)+\frac{b^{2}}{\sinh ^{2} b \rho}-(n-2)^{2} \frac{b^{2}}{\sinh ^{2} b \rho} \\
& \leq-(n-1)^{2} b^{2}+\left(a^{2}-b^{2}\right) .
\end{aligned}
$$

Without loss of generality, we assume $\lambda_{k+1}<\lambda_{k+2}$. By taking $f=\rho$ and $i=1$ in (3-17), we have

$$
\lambda_{k+2}-\lambda_{k+1} \leq 4\left(\lambda_{1}-\frac{1}{4} \int_{\Omega}(\Delta \rho)^{2} u_{1}^{2}-\frac{1}{2} \int_{\Omega}(\nabla(\Delta \rho) \cdot \nabla \rho) u_{1}^{2}\right)^{\frac{1}{2}} \sqrt{\lambda_{k+2}} .
$$

From (4-1) and (4-4), we obtain

$$
\begin{aligned}
\lambda_{1}-\frac{1}{4} \int_{\Omega}(\Delta \rho)^{2} u_{1}^{2}-\frac{1}{2} \int_{\Omega} & (\nabla(\Delta \rho) \cdot \nabla \rho) u_{1}^{2} \\
& =\lambda_{1}+\frac{1}{4} \int_{\Omega}\left(2|\operatorname{Hess} \rho|^{2}+2 \operatorname{Ric}(\nabla \rho, \nabla \rho)-(\Delta \rho)^{2}\right) u_{1}^{2} \\
\leq & \lambda_{1}-\frac{1}{4}(n-1)^{2} b^{2}+\frac{1}{4}\left(a^{2}-b^{2}\right) .
\end{aligned}
$$

By (1-12), (4-5) and (4-6), we have

$$
\begin{aligned}
& \lambda_{k+2}-\lambda_{k+1} \\
& \quad \leq 4\left(\lambda_{1}-\frac{1}{4}(n-1)^{2} b^{2}+\frac{1}{4}\left(a^{2}-b^{2}\right)\right)^{1 / 2} \sqrt{C_{0}(n)\left(\lambda_{1}+\frac{1}{4} n^{2} H_{0}^{2}\right)}(k+1)^{1 / n} \\
& \quad \leq C_{n, \Omega}(k+1)^{1 / n},
\end{aligned}
$$

where $C_{n, \Omega}$ is defined by (1-20). Therefore, we can deduce (4-7) for any $k>1$.

\section{References}

[Andrews and Clutterbuck 2011] B. Andrews and J. Clutterbuck, "Proof of the fundamental gap conjecture”, J. Amer. Math. Soc. 24:3 (2011), 899-916. MR 2012d:35051 Zbl 1222.35130

[Ashbaugh 1999] M. S. Ashbaugh, "Isoperimetric and universal inequalities for eigenvalues", pp. 95139 in Spectral theory and geometry (Edinburgh, 1998), edited by B. Davies and Y. Safarov, London Mathematical Society Lecture Note Series 273, Cambridge University Press, 1999. MR 2001a:35131 Zbl 0937.35114 
[Ashbaugh 2002] M. S. Ashbaugh, "The universal eigenvalue bounds of Payne-Pólya-Weinberger, Hile-Protter, and H. C. Yang”, Proc. Indian Acad. Sci. Math. Sci. 112:1 (2002), 3-30. MR 2004c: 35302 Zbl 1199.35261

[Ashbaugh and Benguria 1989] M. S. Ashbaugh and R. D. Benguria, "Optimal lower bound for the gap between the first two eigenvalues of one-dimensional Schrödinger operators with symmetric singlewell potentials", Proc. Amer. Math. Soc. 105:2 (1989), 419-424. MR 89f:81028 Zbl 0668.34023

[Ashbaugh and Benguria 1991] M. S. Ashbaugh and R. D. Benguria, "Proof of the Payne-PólyaWeinberger conjecture", Bull. Amer. Math. Soc. (N.S.) 25:1 (1991), 19-29. MR 91m:35173 Zbl 0736.35075

[Ashbaugh and Benguria 1992a] M. S. Ashbaugh and R. D. Benguria, "A second proof of the Payne-Pólya-Weinberger conjecture”, Comm. Math. Phys. 147:1 (1992), 181-190. MR 93k:33002 Zbl 0758.34075

[Ashbaugh and Benguria 1992b] M. S. Ashbaugh and R. D. Benguria, "A sharp bound for the ratio of the first two eigenvalues of Dirichlet Laplacians and extensions", Ann. of Math. (2) 135:3 (1992), 601-628. MR 93d:35105 Zbl 0757.35052

[Ashbaugh and Benguria 1993a] M. S. Ashbaugh and R. D. Benguria, "Isoperimetric bounds for higher eigenvalue ratios for the $n$-dimensional fixed membrane problem", Proc. Roy. Soc. Edinburgh Sect. A 123:6 (1993), 977-985. MR 95b:35162 Zbl 0819.35104

[Ashbaugh and Benguria 1993b] M. S. Ashbaugh and R. D. Benguria, "More bounds on eigenvalue ratios for Dirichlet Laplacians in $n$ dimensions", SIAM J. Math. Anal. 24:6 (1993), 1622-1651. MR 94i:35139 Zbl 0809.35067

[Ashbaugh and Benguria 1996] M. S. Ashbaugh and R. D. Benguria, "Bounds for ratios of the first, second, and third membrane eigenvalues", pp. 30-42 in Nonlinear problems in applied mathematics, edited by T. S. Angell et al., Society of Industrial and Applied Mathematics, Philadelphia, 1996. MR 2410595 Zbl 0882.35085

[van den Berg 1983] M. van den Berg, "On condensation in the free-boson gas and the spectrum of the Laplacian”, J. Statist. Phys. 31:3 (1983), 623-637. MR 85i:82022

[Chen and Cheng 2008] D. Chen and Q.-M. Cheng, "Extrinsic estimates for eigenvalues of the Laplace operator", J. Math. Soc. Japan 60:2 (2008), 325-339. MR 2010b:35323 Zbl 1147.35060

[Chen et al. 2012] D. Chen, T. Zheng, and M. Lu, "Eigenvalue estimates on domains in complete noncompact Riemannian manifolds", Pacific J. Math. 255:1 (2012), 41-54. MR 2923693 Zbl 1236.35097

[Cheng and Yang 2005] Q.-M. Cheng and H. Yang, "Estimates on eigenvalues of Laplacian", Math. Ann. 331:2 (2005), 445-460. MR 2005i:58038 Zbl 1122.35086

[Cheng and Yang 2006] Q.-M. Cheng and H. Yang, "Inequalities for eigenvalues of Laplacian on domains and compact complex hypersurfaces in complex projective spaces", J. Math. Soc. Japan 58:2 (2006), 545-561. MR 2007k:58051 Zbl 1127.35026

[Cheng and Yang 2007] Q.-M. Cheng and H. Yang, "Bounds on eigenvalues of Dirichlet Laplacian", Math. Ann. 337:1 (2007), 159-175. MR 2007k:35064 Zbl 1110.35052

[Cheng and Yang 2009] Q.-M. Cheng and H. Yang, "Estimates for eigenvalues on Riemannian manifolds", J. Differential Equations 247:8 (2009), 2270-2281. MR 2010j:58066 Zbl 1180.35390

[El Soufi et al. 2009] A. El Soufi, E. M. Harrell, II, and S. Ilias, "Universal inequalities for the eigenvalues of Laplace and Schrödinger operators on submanifolds”, Trans. Amer. Math. Soc. 361:5 (2009), 2337-2350. MR 2010e:58032 Zbl 1162.58009

[Harrell 1993] E. M. Harrell, II, "Some geometric bounds on eigenvalue gaps", Comm. Partial Differential Equations 18:1-2 (1993), 179-198. MR 94c:35135 Zbl 0810.35067 
[Harrell 2007] E. M. Harrell, II, "Commutators, eigenvalue gaps, and mean curvature in the theory of Schrödinger operators", Comm. Partial Differential Equations 32:1-3 (2007), 401-413. MR 2008i:35041 Zbl 05150097

[Harrell and Michel 1994] E. M. Harrell, II and P. L. Michel, "Commutator bounds for eigenvalues, with applications to spectral geometry", Comm. Partial Differential Equations 19:11-12 (1994), 2037-2055. MR 95i:58182 Zbl 0815.35078

[Harrell and Stubbe 1997] E. M. Harrell, II and J. Stubbe, "On trace identities and universal eigenvalue estimates for some partial differential operators", Trans. Amer. Math. Soc. 349:5 (1997), 1797-1809. MR 97i:35129 Zbl 0887.35111

[Hile and Protter 1980] G. N. Hile and M. H. Protter, "Inequalities for eigenvalues of the Laplacian", Indiana Univ. Math. J. 29:4 (1980), 523-538. MR 82c:35052 Zbl 0454.35064

[Kolmogorov and Fomin 1960] A. N. Kolmogorov and S. V. Fomin, Елементы теории функций и функционального анализа, 2: Мера, интеграл Лебега, Гилбертого пространство, Izdat. Moskov. Univ., Moscow, 1960. Translated as Elements of the theory of functions and functional analysis, 2: Measure. The Lebesgue integral. Hilbert space, Graylock Press, Albany, NY, 1961; reprinted by Dover, Mineola, NY, 1999; Chinese translation, Higher Education Press, Beijing, 2006. MR 0118795 Zbl 0090.08702

[Leung 1991] P.-F. Leung, "On the consecutive eigenvalues of the Laplacian of a compact minimal submanifold in a sphere", J. Austral. Math. Soc. Ser. A 50:3 (1991), 409-416. MR 92d:58212 Zbl 0728.53035

[Li 1980] P. Li, "Eigenvalue estimates on homogeneous manifolds", Comment. Math. Helv. 55:3 (1980), 347-363. MR 81k:58067 Zbl 0451.53036

[Nash 1956] J. Nash, "The imbedding problem for Riemannian manifolds", Ann. of Math. (2) 63 (1956), 20-63. MR 17,782b Zbl 0070.38603

[Payne et al. 1955] L. E. Payne, G. Pólya, and H. F. Weinberger, "Sur le quotient de deux fréquences propres consécutives", C. R. Acad. Sci. Paris 241 (1955), 917-919. MR 17,372d Zbl 0065.08801

[Payne et al. 1956] L. E. Payne, G. Pólya, and H. F. Weinberger, "On the ratio of consecutive eigenvalues", J. Math. and Phys. 35 (1956), 289-298. MR 18,905c Zbl 0073.08203

[Schoen and Yau 1994] R. Schoen and S.-T. Yau, Lectures on differential geometry, Conference Proceedings and Lecture Notes in Geometry and Topology 1, International Press, Cambridge, MA, 1994. MR 97d:53001 Zbl 0830.53001

[Singer et al. 1985] I. M. Singer, B. Wong, S.-T. Yau, and S. S.-T. Yau, "An estimate of the gap of the first two eigenvalues in the Schrödinger operator", Ann. Scuola Norm. Sup. Pisa Cl. Sci. (4) 12:2 (1985), 319-333. MR 87j:35280 Zbl 0603.35070

[Sun et al. 2008] H. Sun, Q.-M. Cheng, and H. Yang, "Lower order eigenvalues of Dirichlet Laplacian”, Manuscripta Math. 125:2 (2008), 139-156. MR 2009i:58042 Zbl 1137.35050

[Thompson 1969] C. J. Thompson, "On the ratio of consecutive eigenvalues in $\mathrm{N}$-dimensions", Studies in Appl. Math. 48 (1969), 281-283. MR 41 \#2242 Zbl 0183.11005

[Weyl 1912] H. Weyl, "Das asymptotische Verteilungsgesetz der Eigenwerte linearer partieller Differentialgleichungen (mit einer Anwendung auf die Theorie der Hohlraumstrahlung)", Math. Ann. 71:4 (1912), 441-479. MR 1511670 JFM 43.0436.01

[Wu et al. 1989] H. Wu, L. C. Shen, and Y. L. Yu, Introduction to Riemannian geometry, Peking University Press, Beijing, 1989. In Chinese.

[Yang 1991] H. Yang, "An estimate of the difference between consecutive eigenvalues", preprint, International Centre for Theoretical Physics, Trieste, 1991, available at https://inis.iaea.org/search/ search.aspx?orig_q=RN:23015356. Report IC/91/60. 
[Yang and Yau 1980] P. C. Yang and S.-T. Yau, "Eigenvalues of the Laplacian of compact Riemann surfaces and minimal submanifolds", Ann. Scuola Norm. Sup. Pisa Cl. Sci. (4) 7:1 (1980), 55-63. MR 81m:58084 Zbl 0446.58017

[Yau 1986] S.-T. Yau, Nonlinear analysis in geometry, Monographies de L'Enseignement Mathématique 33, L'Enseignement Mathématique, Geneva, 1986. MR 88e:53001 Zbl 0631.53003

[Yu and Zhong 1986] Q. H. Yu and J. Q. Zhong, "Lower bounds of the gap between the first and second eigenvalues of the Schrödinger operator", Trans. Amer. Math. Soc. 294:1 (1986), 341-349. MR 87f:35179 Zbl 0593.53030

[Zeidler 1995] E. Zeidler, Applied functional analysis: main principles and their applications, Applied Mathematical Sciences 109, Springer, New York, 1995. MR 96i:00006 Zbl 0834.46003

Received November 17, 2014.

DAGUANG CHEN

DEPARTMENT OF MATHEMATICAL SCIENCES

TSINGHUA UNIVERSITY

BEIJING 100084

CHINA

dgchen@math.tsinghua.edu.cn

TAO ZHENG

SCHOOL OF MATHEMATICS AND STATISTICS

BEIJING INSTITUTE OF TECHNOLOGY

BEIJING 100081

CHINA

zhengtao08@amss.ac.cn

HONGCANG YANG

Hua Loo-Keng Key Laboratory of Mathematics

CHINESE ACADEMY OF SCIENCES

BEIJING 100080

CHINA

yanghc2@netease.com 


\title{
PACIFIC JOURNAL OF MATHEMATICS
}

\author{
msp.org/pjm
}

Founded in 1951 by E. F. Beckenbach (1906-1982) and F. Wolf (1904-1989)

\section{EDITORS}

Don Blasius (Managing Editor)

Department of Mathematics

University of California

Los Angeles, CA 90095-1555

blasius@math.ucla.edu

\author{
Paul Balmer \\ Department of Mathematics \\ University of California \\ Los Angeles, CA 90095-1555 \\ balmer@math.ucla.edu \\ Robert Finn \\ Department of Mathematics \\ Stanford University \\ Stanford, CA 94305-2125 \\ finn@math.stanford.edu \\ Sorin Popa \\ Department of Mathematics \\ University of California \\ Los Angeles, CA 90095-1555 \\ popa@math.ucla.edu
}

\author{
Vyjayanthi Chari \\ Department of Mathematics \\ University of California \\ Riverside, CA 92521-0135 \\ chari@math.ucr.edu \\ Kefeng Liu \\ Department of Mathematics \\ University of California \\ Los Angeles, CA 90095-1555 \\ liu@math.ucla.edu \\ Jie Qing \\ Department of Mathematics \\ University of California \\ Santa Cruz, CA 95064 \\ qing@ cats.ucsc.edu
}

\section{PRODUCTION}

Silvio Levy, Scientific Editor, production@msp.org

\section{SUPPORTING INSTITUTIONS}

ACADEMIA SINICA, TAIPEI

CALIFORNIA INST. OF TECHNOLOGY

INST. DE MATEMÁTICA PURA E APLICADA

KEIO UNIVERSITY

MATH. SCIENCES RESEARCH INSTITUTE

NEW MEXICO STATE UNIV.

OREGON STATE UNIV.

\author{
STANFORD UNIVERSITY \\ UNIV. OF BRITISH COLUMBIA \\ UNIV. OF CALIFORNIA, BERKELEY \\ UNIV. OF CALIFORNIA, DAVIS \\ UNIV. OF CALIFORNIA, LOS ANGELES \\ UNIV. OF CALIFORNIA, RIVERSIDE \\ UNIV. OF CALIFORNIA, SAN DIEGO \\ UNIV. OF CALIF., SANTA BARBARA
}

\author{
Daryl Cooper \\ Department of Mathematics \\ University of California \\ Santa Barbara, CA 93106-3080 \\ cooper@math.ucsb.edu \\ Jiang-Hua Lu \\ Department of Mathematics \\ The University of Hong Kong \\ Pokfulam Rd., Hong Kong \\ jhlu@maths.hku.hk \\ Paul Yang \\ Department of Mathematics \\ Princeton University \\ Princeton NJ 08544-1000 \\ yang@math.princeton.edu
}

These supporting institutions contribute to the cost of publication of this Journal, but they are not owners or publishers and have no responsibility for its contents or policies.

See inside back cover or msp.org/pjm for submission instructions.

The subscription price for 2016 is US $\$ 440 /$ year for the electronic version, and $\$ 600 /$ year for print and electronic.

Subscriptions, requests for back issues and changes of subscriber address should be sent to Pacific Journal of Mathematics, P.O. Box 4163, Berkeley, CA 94704-0163, U.S.A. The Pacific Journal of Mathematics is indexed by Mathematical Reviews, Zentralblatt MATH, PASCAL CNRS Index, Referativnyi Zhurnal, Current Mathematical Publications and Web of Knowledge (Science Citation Index).

The Pacific Journal of Mathematics (ISSN 0030-8730) at the University of California, c/o Department of Mathematics, 798 Evans Hall \#3840, Berkeley, CA 94720-3840, is published twelve times a year. Periodical rate postage paid at Berkeley, CA 94704, and additional mailing offices. POSTMASTER: send address changes to Pacific Journal of Mathematics, P.O. Box 4163, Berkeley, CA 94704-0163.

PJM peer review and production are managed by EditFLOW ${ }^{\circledR}$ from Mathematical Sciences Publishers.

\section{PUBLISHED BY}

\section{mathematical sciences publishers \\ nonprofit scientific publishing}

http://msp.org/

(C) 2016 Mathematical Sciences Publishers 


\section{PACIFIC JOURNAL OF MATHEMATICS}

Volume $282 \quad$ No. $2 \quad$ June 2016

Exhausting curve complexes by finite rigid sets

JAVIER ARAMAYONA and CHRISTOPHER J. LEININGER

A variational characterization of flat spaces in dimension three

Giovanni Catino, Paolo Mastrolia and Dario D. Monticelli

Estimates of the gaps between consecutive eigenvalues of Laplacian

293

DAgUang Chen, TAO Zheng and HongCANG YANG

Liouville type theorems for the $p$-harmonic functions on certain manifolds

JINGYI CHEN and YUE WANG

Cartan-Fubini type rigidity of double covering morphisms of quadratic manifolds

\section{HOSUNG KIM}

On the uniform squeezing property of bounded convex domains in $\mathbb{C}^{n}$

KANG-TAE KIM and LIYOU ZHANG

Lefschetz pencils and finitely presented groups

RYOMA KOBAYASHI and NAOYUKI MONDEN

Knot homotopy in subspaces of the 3-sphere

YUYA KODA and MAKOTO OzAWA

On the relationship of continuity and boundary regularity in prescribed mean curvature Dirichlet problems

KIRK E. LANCASTER and JARON MELIN

Bridge spheres for the unknot are topologically minimal

JUNG HOON LEE

On the geometric construction of cohomology classes for cocompact discrete subgroups of $\mathrm{SL}_{n}(\mathbb{R})$ and $\mathrm{SL}_{n}(\mathbb{C})$

SUSANNE SCHIMPF

On Blaschke's conjecture

Xiaole Su, Hongwei Sun and Yusheng Wang

The role of the Jacobi identity in solving the Maurer-Cartan structure equation 